\title{
TAGUNG
}

\section{„Strong Europe with a Human Touch" - Erwartungen an die erste ungarische Ratspräsidentschaft}

\author{
Tamás Szigetvári, Gábor Túry und Krisztina Vida*
}

Das Institut für Weltwirtschaft an der Ungarischen Akademie der Wissenschaften hat zwischen Ende November und Mitte Dezember 2010, im Vorfeld der ungarischen Ratspräsidentschaft, drei internationale Konferenzen veranstaltet. Alle drei waren Teil eines Projektes zur wissenschaftlichen Vorbereitung der Ratspräsidentschaft. Im Rahmen des Vorhabens wurden kontinuierlich Analysen durchgeführt und im Spätsommer eine Evaluierung der ungarischen Vorbereitungen im Rahmen einer internationalen Konferenz vorgenommen.

Hauptthemen der Konferenzen waren der allgemeine Stand der europäischen Integration mit Blick auf das Jahr 2011 und auf mittlere Sicht, die Erwartungen hinsichtlich der ungarischen Ratspräsidentschaft und die - mehreren Veränderungen ausgesetzten - ungarischen Prioritäten zu überprüfen.

Der ungarische Vorsitz im Lichte der Erfahrungen junger Mitgliedstaaten und der gegenwärtigen Entwicklungen in der $E U^{l}$

Die erste Tagung hat Experten aus Nichtregierungsorganisationen der neuen mittel-osteuropäischen Mitgliedstaaten zusammengebracht. Die für Journalisten nicht zugängliche Konferenz, die im Institut für Weltwirtschaft,
Budapest, veranstaltet wurde, widmete sich drei Kernbereichen. Erstens wurden die Erfahrungen von zwei neuen Mitgliedstaaten (Slowenien und Tschechien) mit der Ratspräsidentschaft erörtert sowie die Vorbereitungen von Ungarn und Polen auf die aufeinanderfolgenden Ratspräsidentschaften im Jahr 2011 vorgestellt. Im zweiten Block legten die Experten aus den einzelnen neuen Mitgliedstaaten ihre Sicht und Überlegungen hinsichtlich der zu erwartenden (und teilweise der ,überraschenden') Entwicklungen in der Europäischen Union dar. Das dritte Thema umfasste die eigenen Prioritäten und Erwartungen der neuen Mitgliedstaaten an die ungarische Ratspräsidentschaft.

Petr Drulák fasste die wichtigsten Erfahrungen der slowenischen und der tschechischen Ratspräsidentschaft ${ }^{2}$ zusammen. Er unterstrich die Bedeutung innenpolitischer Stabilität als Voraussetzung einer erfolgreichen Ratspräsidentschaft, verbunden mit effizienten Institutionen, zielgerichteter Kommunikation und einem echten Dialog mit der Zivilgesellschaft. Darüber hinaus hänge der Erfolg auch davon ab, wie der vorsitzende Mitgliedstaat mit den beiden großen und führenden Mächten, namentlich Deutschland und Frankreich, zusammenarbeitet. Um die Prioritäten

* Tamás Szigetvári, PhD, Wissenschaftlicher Mitarbeiter, Institut für Weltwirtschaft, Ungarische Akademie der Wissenschaften, Budapest.

Gábor Túry, MSC, Wissenschaftlicher Mitarbeiter, Institut für Weltwirtschaft, Ungarische Akademie der Wissenschaften, Budapest.

Krisztina Vida, PhD, Wissenschaftliche Mitarbeiterin, Institut für Weltwirtschaft, Ungarische Akademie der Wissenschaften, Budapest.

1 Das Programm der Tagung ist abrufbar unter: http://archiv.zeitschrift-integration.de/Tagungsprogramme_12011.pdf (letzter Zugriff: 16.02.2011).

2 Er stützte sich bei seinen Ausführungen auf den soeben erschienenen Band von Petr Drulák/Zlatko Šabiě (Hrsg.): The Czech and Slovenian EU presidencies in a comparative perspective, Dordrecht 2010. 
eines mittleren oder kleinen Mitgliedstaates wenigstens teilweise verwirklichen zu können, benötigten diese Staaten Partner, die sich der Zukunft der europäischen Integration verpflichtet fühlen. Andrej Kumar setzte diesen Gedankengang fort, indem er betonte, dass ein kleiner Mitgliedstaat kaum Chancen auf eine ,high-profile'-Ratspräsidentschaft habe. Trotzdem gebe es einige Bereiche, in denen auch kleinere Staaten einen positiven Beitrag leisten könnten. In dieser Hinsicht machte er auf die gemeinsame geografische Lage und politisch-wirtschaftliche Interessen von Slowenien und Ungarn am westlichen Balkan im Allgemeinen und an der Unterstützung der Integration dieser Region in die Europäische Union im Besonderen aufmerksam.

Alle Experten wiesen darauf hin, dass ein neues Halbjahr der Ratspräsidentschaft, insbesondere im Rahmen der Trio-Präsidentschaften (derzeit Spanien/Belgien/Ungarn und danach Polen/Zypern/Dänemark), den Spielraum, eigene Prioritäten zu setzen, einenge. Es gebe nämlich eine Reihe von Angelegenheiten, die von der vorhergehenden Ratspräsidentschaft geerbt werden. Schon deshalb seien Kontinuität und ein intensiver Dialog zwischen den einzelnen Ratspräsidentschaften unerlässlich. So habe die teilweise Unterbrechung der Kommunikation und die Manifestierung unterschiedlicher Zielkonflikte den Spielraum Tschechiens erheblich eingeschränkt. Auch Elżbieta Kaca hob die Notwendigkeit eines umfassenden Dialogs hervor. Dabei wolle Polen seine Ratspräsidentschaft auch dazu nutzen, die Bedeutung der mitteleuropäischen Region für die weitere Entwicklung der europäischen Integration zu unterstreichen.

Alle Experten sahen die grundlegenden Herausforderungen der Europäischen Union in der Zukunft der Eurozone, der Schaffung beziehungsweise Erhöhung der globalen Wettbewerbsfähigkeit der Europäischen Union, der ,Lösung ‘ gesellschaftlicher und ethnischer Konflikte sowie in erfolgreichen Rezepten zur Reduzierung der nationalen Haus- halts- und Staatsdefizite. Rumyana Kolarova hielt die gesellschaftliche Unterstützung für gemeinsame Programme für ebenso wichtig wie die Formulierung von Programmen mit konkreten Zielen und Instrumenten. Sie betonte, dass ohne den Bedeutungsverlust der Politik zu stoppen, nicht mit wirksamer sozialer Unterstützung gerechnet werden könne. Die einzelnen Positionen und Prioritäten der Ratspräsidentschaft gingen zwar in mehreren Fällen auseinander, doch kristallisiere sich ein gemeinsamer europäischer Standpunkt heraus. Der Aufholprozess der neuen Mitgliedstaaten hänge eng mit einer effizienten und global wettbewerbsfähigen Europäischen Union zusammen. Darüber hinaus betrachteten die Baltischen Staaten - auch aus sicherheitspolitischen Überlegungen - die europäische Integration und ihren eigenen erfolgreichen Aufholprozess als ein Gegengewicht zu russischen Hegemoniebestrebungen. Sowohl Erik Terk als auch Ram̃unas Vilpišauskas betonten die Notwendigkeit, eines global wettbewerbsfähigen Europas. Dazu sei die konsequente Fortführung (oder Einführung) grundlegender Reformen sowie die Vertiefung der Integration unvermeidbar. Auch András Inotai teilte diese Auffassung und betonte die Wichtigkeit der finanziellen Stabilisierung der Europäischen Union (und insbesondere der Eurozone).

Im Zusammenhang mit den Erwartungen an die ungarische Ratspräsidentschaft standen wieder einmal die wirtschaftlichen und wettbewerbsbezogenen Herausforderungen im Vordergrund. Der Abschluss der Beitrittsverhandlungen mit Kroatien, die als Priorität betrachtete Donau-Strategie sowie die Verstärkung der Östlichen Partnerschaft könnten einen wichtigen Beitrag zur weiteren Offenheit der Europäischen Union leisten. Ivo Šlosarčík unterbreitete konkrete Vorschläge für die ungarische Ratspräsidentschaft. Ihm zufolge soll die ungarische Ratspräsidentschaft nicht unsichtbar, nicht konfliktproduzierend und nicht zuletzt keine Wiederholung der tschechischen Ratspräsidentschaft sein, die die Periode der Ratspräsidentschaft ein- 
fach ,überleben“ wollte. Im Gegensatz werde eine Ratspräsidentschaft mit Entscheidungsfähigkeit erwartet, die konkrete Lösungsvarianten in der europäischen Energiepolitik formuliert, zur Erneuerung der Gemeinsamen Außen- und Sicherheitspolitik beiträgt und trotz des engen Spielraums - eine aktive Rolle in der Gestaltung der Außenbeziehungen der Europäischen Union einnimmt.

András Inotai bewertete in seinen abschlieBenden Bemerkungen die Konferenz als eindeutig erfolgreich. Die Teilnehmer hätten ihrer Hoffnung Ausdruck gegeben, dass dieser Kreis auch in der Zukunft - sowohl während als auch nach der ungarischen Ratspräsidentschaft - eine fachlich hochwertige und etablierte Grundlage für die weitere gemeinsame Analyse der wichtigsten Probleme der europäischen Integration mit Blick auf die Herausforderungen und Lösungsvarianten in den neuen Mitgliedstaaten bieten werde. ${ }^{3}$

Die TEPSA Pre-Presidency Conference: Prioritäten der Präsidentschaft und Ansichten der EU-Partner ${ }^{4}$

Diese Veranstaltung stand in der jahrzehntelangen Tradition der Transeuropean Policy Studies Association (TEPSA), regelmäßig eine „Pre-Presidency Conference“ in dem Mitgliedstaat zu organisieren, der im jeweils nächsten Halbjahr die Ratspräsidentschaft übernimmt. Als langjähriges ungarisches Mitglied von TEPSA nahm diese Aufgabe das Institut für Weltwirtschaft wahr. Teilnehmer der Konferenz waren Vertreter der Mitgliedsinstitutionen von TEPSA sowie von THESEUS ${ }^{5}$, die Ungarische Vertretung der Europäischen Kommission sowie viele Experten und Interessierte aus der ungarischen akademischen und Hochschulwelt.
Unter dem Titel „Entering the new decade: New challenges and priorities of the EU under the Hungarian Council Presidency" war die Konferenz in zwei Plenarsitzungen und sieben teilweise parallel laufenden Sektionen organisiert. Die Konferenz wurde von András Inotai und Jean-Paul Jacqué eröffnet. Ausgangspunkt der Konferenz waren die „TEPSA Recommendations” zur ungarischen Ratspräsidentschaft. Anschließend hob ein Vertreter der ungarischen Regierung fünf Kernaufgaben der kommenden Ratspräsidentschaft hervor: das Europäische Semester in Verbindung mit der Europa 2020-Strategie umzusetzen, Unterstützung für die Eurozone und andere gemeinsame Politiken (beispielsweise das Politikfeld Energie) zu leisten, die Umsetzung des Stockholmer Programms fortzuführen und das Europa der Bürger zu stärken, den Erweiterungsprozess zu beschleunigen (Beitritt Kroatiens, Schengen-Beitritt Bulgariens und Rumäniens sowie neue Impulse für den Westbalkan) und die Östliche Partnerschaft zu stärken, mit dem ungarischen Leitmotiv „enlarging responsibly and engaging globally“. Im weiteren Verlauf der ersten Plenarsitzung sprachen die Botschafter von Spanien und Belgien über die Erfahrungen und Errungenschaften im Rahmen der jeweiligen Ratspräsidentschaft sowie die der ungarischen Ratspräsidentschaft überlassenen Aufgaben. Péter Balázs hob konkrete Herausforderungen und Chancen der ungarischen Ratspräsidentschaft hervor, mit besonderem Nachdruck die Krise der Eurozone und das Anlaufen der DonauStrategie. Tamás Szúcs sowie Ádám Török betonten die Notwendigkeit der Verstärkung der globalen Wettbewerbsfähigkeit der Europäischen Union.

Die Sektionen beschäftigten sich mit den folgenden Themen: Bewertung der bisherigen

3 Die Beiträge der Konferenz werden im ersten Quartal 2011 in einem Sammelband veröffentlicht.

4 Das Programm der Tagung ist abrufbar unter: http://archiv.zeitschrift-integration.de/Tagungsprogramme_12011.pdf (letzter Zugriff: 16.02.2011).

5 Das Forschungsprogramm „THESEUS - eine europäische Gesellschaft schaffen“ wird gemeinsam vom Forschungsinstitut für Politische Wissenschaft und Europäische Fragen der Universität zu Köln, dem Institut d'études politiques, Paris, der Trans European Policy Studies Association, Brüssel, und der Fritz Thyssen Stiftung getragen. 
Umsetzung des Vertrags von Lissabon; Gegenwart und Zukunft der Eurozone; die Perspektive der Erweiterung und die Östliche Partnerschaft; die Europa 2020-Strategie; der neue Finanzrahmen ab 2014; die Donau-Strategie; sowie Europa der Bürger. In der ersten Sektion wurde unterstrichen, dass der Vertrag von Lissabon die Rolle des Staats- oder Regierungschefs des Staates, der die Ratspräsidentschaft inne hat, erheblich einschränkt. Positiv wurde der mögliche Beitrag des Vertrags von Lissabon zur gemeinsamen Außen-, Sicherheits- und Verteidigungspolitik der Europäischen Union gesehen, auch im Hinblick auf eine bessere Koordinierung der EU-Mitgliedstaaten innerhalb der NATO. Der neu etablierte Europäische Auswärtige Dienst biete eine Chance zur besseren Koordinierung der intergouvernementalen und gemeinschaftlichen Ansätze und könne eine positive Sozialisierungswirkung auf die beteiligten Diplomaten aus den einzelnen Mitgliedstaaten ausüben.

Nach einer eingehenden Analyse der Ursachen der gegenwärtigen Krise der Eurozone, einschließlich der ,Lücken“ der fiskalischen Koordination, der großen strukturellen Unterschiede zwischen den einzelnen Mitgliedstaaten sowie der Geburtsfehler der Währungsunion, wurde der ,bail-out ${ }^{\star}$ einzelner Mitgliedstaaten kontrovers diskutiert. Die Teilnehmer stimmten darin überein, dass die Einführung und konsequente Durchsetzung neuer und strengerer Maßnahmen geboten sei.

Die Erweiterungssektion begann mit Berichten aus den drei potenziellen Mitgliedstaaten, Kroatien, der Türkei und Island. Die Unterschiede zwischen den Beitrittsperspektiven der drei Staaten sowie den Interessen an einem Beitritt kamen klar zum Ausdruck. Die Experten unterstrichen die Wichtigkeit der Östlichen Partnerschaft, deren nächstes Gipfeltreffen im Mai 2011 in Ungarn stattfinden wird.

Die Arbeitsgruppe zur Europa 2020-Strategie setzte sich kritisch mit den Zielen, Entschei- dungsmechanismen und tatsächlichen Möglichkeiten der Strategie auseinander. Einigkeit herrschte jedoch in der Frage, dass - unabhängig von irgendeiner Strategie - der Schlüsselfaktor für eine Verbesserung der europäischen Wettbewerbsfähigkeit die Erhöhung der Produktivität sei.

Zwar wird die offizielle Debatte über den neuen Finanzrahmen (ab 2014) erst während der polnischen Ratspräsidentschaft beginnen, doch kann Ungarn im ersten Halbjahr 2011 wichtige informelle Gespräche initiieren, um so die derzeit recht weit auseinanderklaffenden Positionen der Mitgliedstaaten einander näherzubringen. Eine (erhebliche) Erhöhung des gemeinsamen Finanzrahmens wurde von niemandem vorgeschlagen. Vielmehr ging die Debatte um neue Prioritäten und die Umstrukturierung der zu finanzierenden Bereiche.

Die Arbeitsgruppe über die Donau-Strategie befasste sich nicht nur mit den potenziellen Vorteilen, sondern auch mit den Mängeln und offenen Fragen dieser Initiative. Es wurde festgestellt, dass sich eine makroregionale $\mathrm{Zu}$ sammenarbeit, die die Donau-Strategie erfordert und teilweise voraussetzt, auf keine Erfahrung oder Tradition bauen kann. Ähnlich der baltischen Region gibt es vielmehr meistens vielversprechende Pläne, aber noch keine konkreten Projektvorschläge.

Die siebte Gruppe beschäftigte sich mit dem Konzept „Europa der Bürger“ im Kontext der Entwicklung des Raums der Freiheit, der Sicherheit und des Rechts, unter besonderer Betonung der Umsetzung des Stockholmer Programms.

Die zweite Plenarsitzung begann mit der Berichterstattung aus den Arbeitsgruppen, gefolgt von einer abschließenden Bewertung durch András Inotai und Wolfgang Wessels, die die wichtigsten Ergebnisse der Konferenz im Hinblick auf die ungarische Ratspräsidentschaft zusammenfassten. Die herrschende Meinung war, dass Ungarn diese Rolle in einer kritischen Phase der europäischen Integra- 
tion übernimmt. Das Programm der ungarischen Ratspräsidentschaft sei ambitiös aber realistisch.

Im Rahmen der Konferenz wurde der „THESEUS Award for Promising Research on European Integration" an Mareike Kleine und an Virginie Van Ingelgom verliehen. Helen Wallace erhielt den ,THESEUS Award for Outstanding Research on European Integration“.

Die Östliche Partnerschaft und Erwartungen an die ungarische Ratspräsidentschaft ${ }^{6}$

Die Konferenz mit Experten aus den Staaten der Östlichen Partnerschaft widmete sich den beiden folgenden Hauptthemen. Erstens, wie sehen die Experten aus diesen Ländern kurzund mittelfristig die Entwicklungen in der Europäischen Union und deren Folgen für die bilateralen Beziehungen. Zweitens, was erwarten sie von der ungarischen Ratspräsidentschaft, die das zweite Gipfeltreffen der Östlichen Partnerschaft ausrichten wird.

Zunächst wurde die offizielle Terminologie „Östliche Partnerschaft” diskutiert. Den Teilnehmern zufolge sollte diese Bezeichnung in „europäische Partnerschaft” geändert werden, um die Gegenseitigkeit der Beziehungen besser zum Ausdruck bringen zu können. Anschließend befasste man sich mit den eigentlichen Inhalten der Partnerschaft, insbesondere ob sie die Vorreiterin einer zukünftigen EUMitgliedschaft ist oder nicht. Die Auffassungen der Teilnehmer gingen in dieser Frage weit auseinander, allein über die Wichtigkeit der Östlichen Partnerschaft herrschte Einigkeit. Das ,Eastern Partnership - Civil Society Forum" in Berlin habe gezeigt, dass nicht unbedingt die Regierungen und staatliche Institutionen der Motor dieses Prozesses sein müssen, sondern die Zivilgesellschaften diese Rolle immer mehr übernehmen kann.
Wie erwartet, wurde der Frage der Visaliberalisierung besondere Aufmerksamkeit geschenkt. In diesem Ziel oder wenigstens in der erheblichen Vereinfachung der Einreisemöglichkeiten in die EU-Mitgliedstaaten würden die meisten Staaten der Östlichen Partnerschaft ein wichtiges Symbol für die weitere Annäherung sehen.

Besonders von ukrainischer Seite wurde die Wichtigkeit eines umfassenden Freihandelsabkommens unterstrichen, das nicht nur wichtige Elemente des einheitlichen Binnemarktes (Rechtsharmonisierung) einschließt, sondern auch zahlreiche Agrarprodukte umfassen sollte. Weiterhin wurde die Aufstockung der finanziellen Mittel seitens der Europäischen Union hervorgehoben.

Mehrere Teilnehmer waren der Auffassung, dass die Europäische Union eine wichtige Rolle bei der Lösung der ,eingefrorenen Konflikte" (zwischen Armenien und Aserbaidschan, zwischen Georgien und Russland, aber auch in Transnistrien) spielen sollte. In diesem Zusammenhang dürfe man die geopolitischen Realitäten, nämlich das Vorhandensein teilweise starker russischer Interessen und Verflechtungen mit ausgewählten Staaten der Östlichen Partnerschaft, nicht aus den Augen verlieren. Schon deshalb könne die Östliche Partnerschaft nicht von der allgemeinen Entwicklung der Beziehungen zwischen der Europäischen Union und Russland getrennt werden.

Die recht offene Diskussion der Teilnehmer verdeutlichte, dass die politischen und wirtschaftlichen Bedingungen (und Interessen) der einzelnen Partnerstaaten, einschließlich ihrer Beziehungen zur Europäischen Union, teilweise erhebliche Unterschiede zutage bringen. Mit Blick auf die innenpolitischen Verhältnisse sind Armenien, Aserbaidschan und Belarus als autoritäre Regime zu klassifizieren, während Georgien, Moldova und die

6 Das Programm der Tagung ist abrufbar unter: http://archiv.zeitschrift-integration.de/Tagungsprogramme_12011.pdf (letzter Zugriff: 16.02.2011). 
Ukraine sich durch demokratischere Institutionen auszeichnen. Nicht weniger unterschiedlich seien die wirtschaftlichen Entwicklungen und die weiteren Aussichten. Aserbaidschan benötige zum Beispiel kaum finanzielle Unterstützung von der Europäischen Union. Dies bedeute jedoch, dass die Europäische Union auch weniger Einfluss auf die Demokratisierungsprozesse in diesem Land nehmen kann. Doch dürfe die Forderung nach mehr Demokratie nicht zugunsten europäischer Interessen an Energieversorgungssicherheit preisgegeben werden.

Die Teilnehmer formulierten konkrete Empfehlungen für die ungarische Ratspräsidentschaft. Erstens solle der zweite Gipfel der Östlichen Partnerschaft pragmatische Ergebnisse bringen, im Vergleich zum eher deklaratorischen ersten Gipfel in Prag vor zwei Jahren. Zweitens soll die Europäische Union die finanzielle Unterstützung mit den bedeutenden politischen Zielen in Einklang bringen. Drittens sollen die einzelnen Staaten der Östlichen Partnerschaft eine ,Messlatte" erhalten, an der sie ihre eigenen Fortschritte auch im
Vergleich zu den anderen Staaten der Region messen können. Viertens erwarten die meisten Staaten eine aktivere Rolle der Europäischen Union bei regionalen Sicherheitsfragen und in der konkreten Konfliktbehandlung. Schliesslich solle mehr Aufmerksamkeit der Zivilgesellschaft gewidmet werden, die zur Transparenz der beiderseitigen Beziehungen einen konstruktiven Beitrag leisten kann.

Die drei Konferenzen des Budapester Instituts für Weltwirtschaft über die vielschichtigen Themen der ungarischen Ratspräsidentschaft wurden von vielen ungarischen wie internationalen Teilnehmern besucht, deren Vorträge lebhafte Debatten provozierten. Das Institut für Weltwirtschaft engagiert sich auch im ersten Halbjahr 2011 weiterhin intensiv in der wissenschaftlichen Begleitung der ungarischen Ratspräsidentschaft und sucht dazu den Austausch mit den relevanten internationalen politischen und wirtschaftlichen Akteuren. Begleitend werden auf der Internetseite des Instituts regelmäßig kurze Analysen in englischer Sprache veröffentlicht sowie weitere Diskussionsrunden und Konferenzen organisiert.

\title{
Korruptionsbekämpfung im postsozialistischen Bulgarien
}

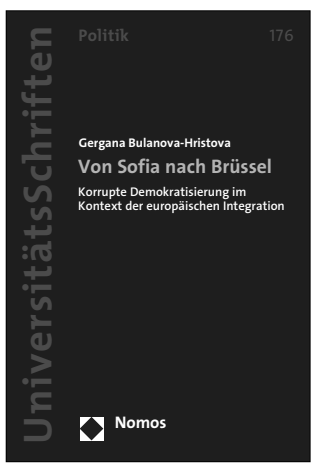

\author{
Von Sofia nach Brüssel \\ Korrupte Demokratisierung im Kontext \\ der europäischen Integration \\ Von Gergana Bulanova-Hristova
}

2011, 433 S., brosch., 69,- €, ISBN 978-3-8329-6037-7

(Nomos Universitätsschriften - Politik, Bd. 176)

Bitte bestellen Sie im Buchhandel oder versandkostenfrei unter $\boldsymbol{}$ www.nomos-shop.de

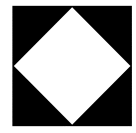

Nomos 